\title{
The PMIP4 Last Glacial Maximum experiments: preliminary results and comparison with the PMIP3 simulations
}

Masa Kageyama et al.

Correspondence to: Masa Kageyama (masa.kageyama@1sce.ipsl.fr)

The copyright of individual parts of the supplement might differ from the article licence. 


\section{Supplementary material}

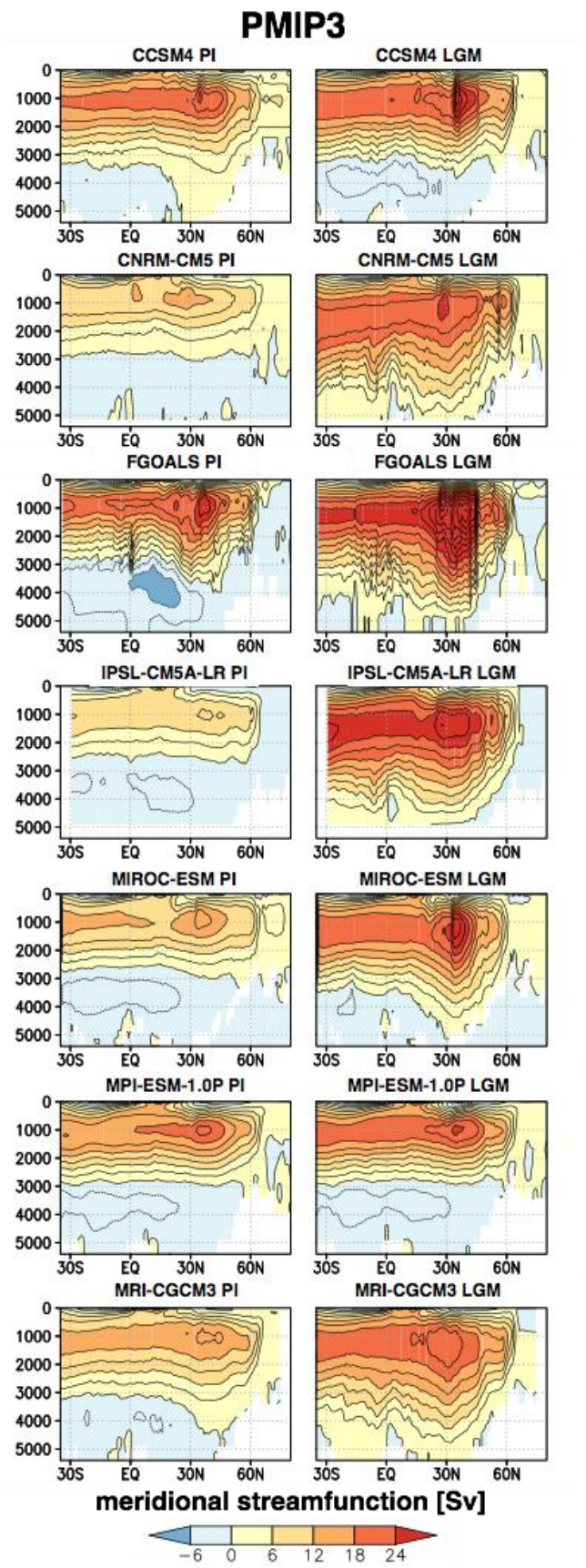

Supplementary Figure S1: Atlantic Meridional Streamfunction (Sv) in the PMIP3 PI and LGM experiments, for the models for which the data was available. 
PMIP4
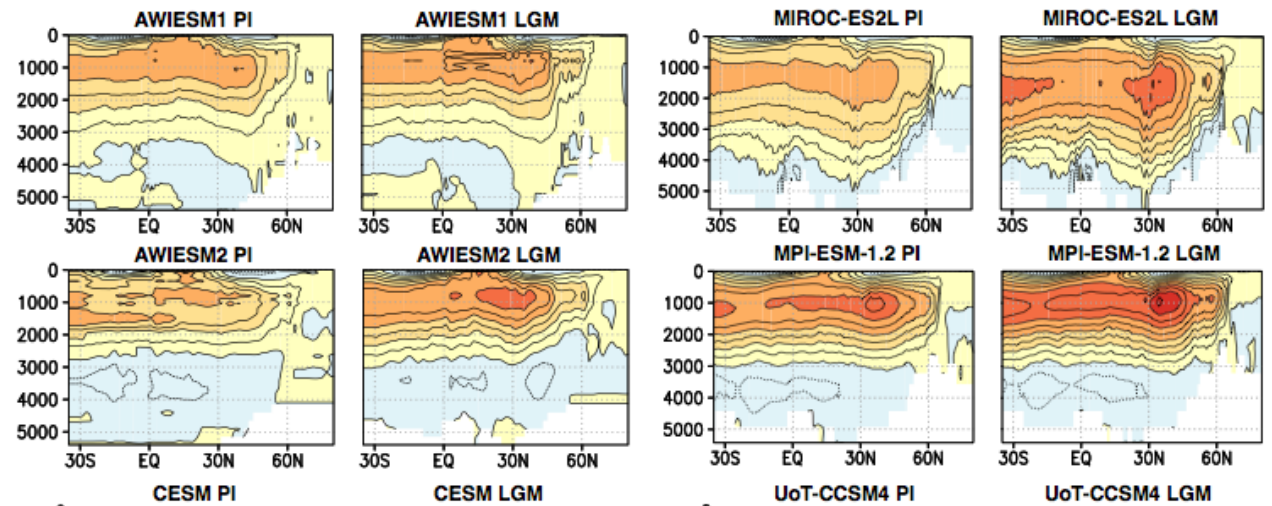

MPI-ESM-1.2 LGM
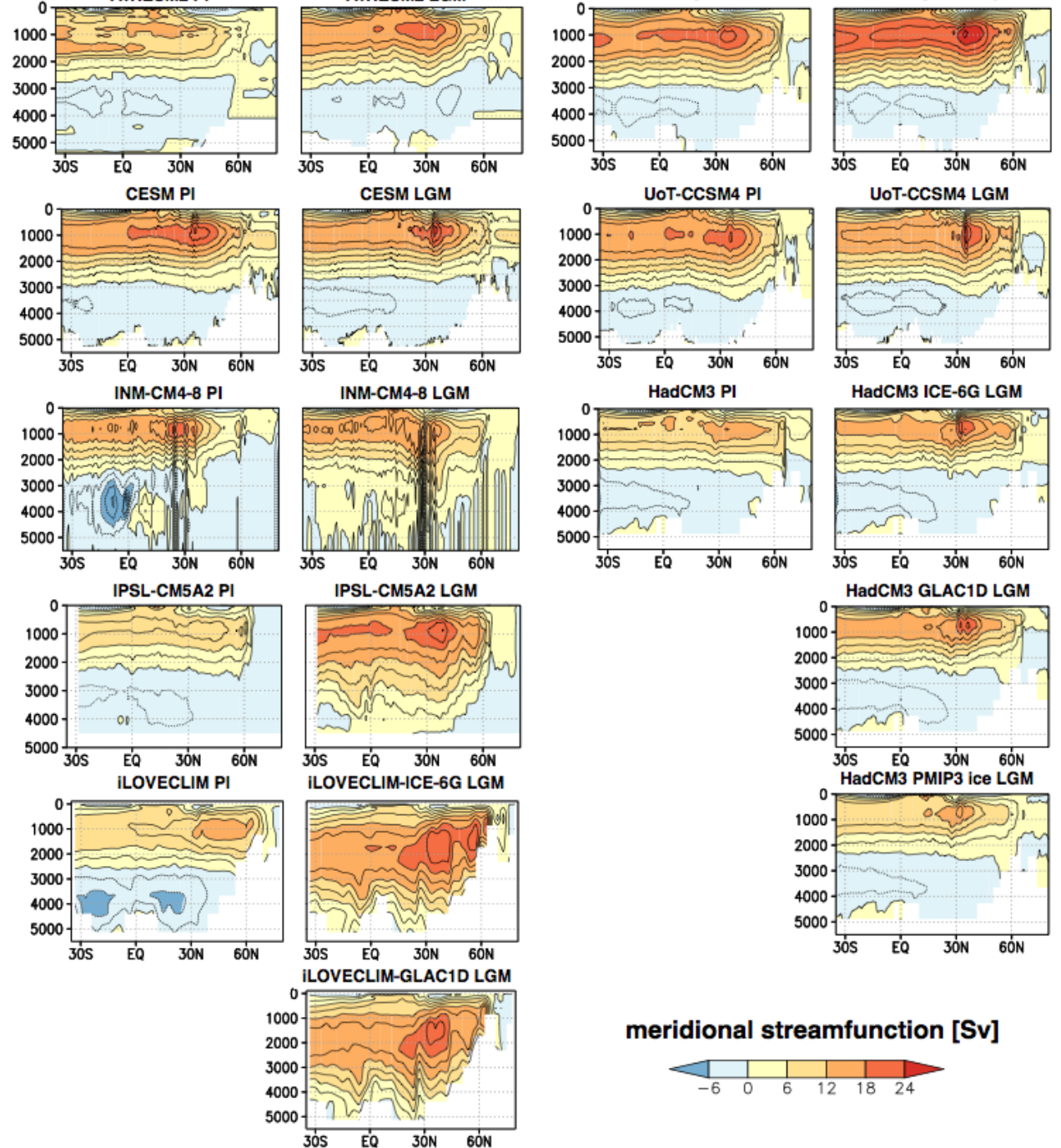

\section{meridional streamfunction [Sv]}

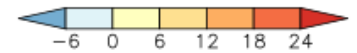

Supplementary Figure S2: Atlantic Meridional Streamfunction (Sv), for the PI and LGM, for the PMIP4 models. 

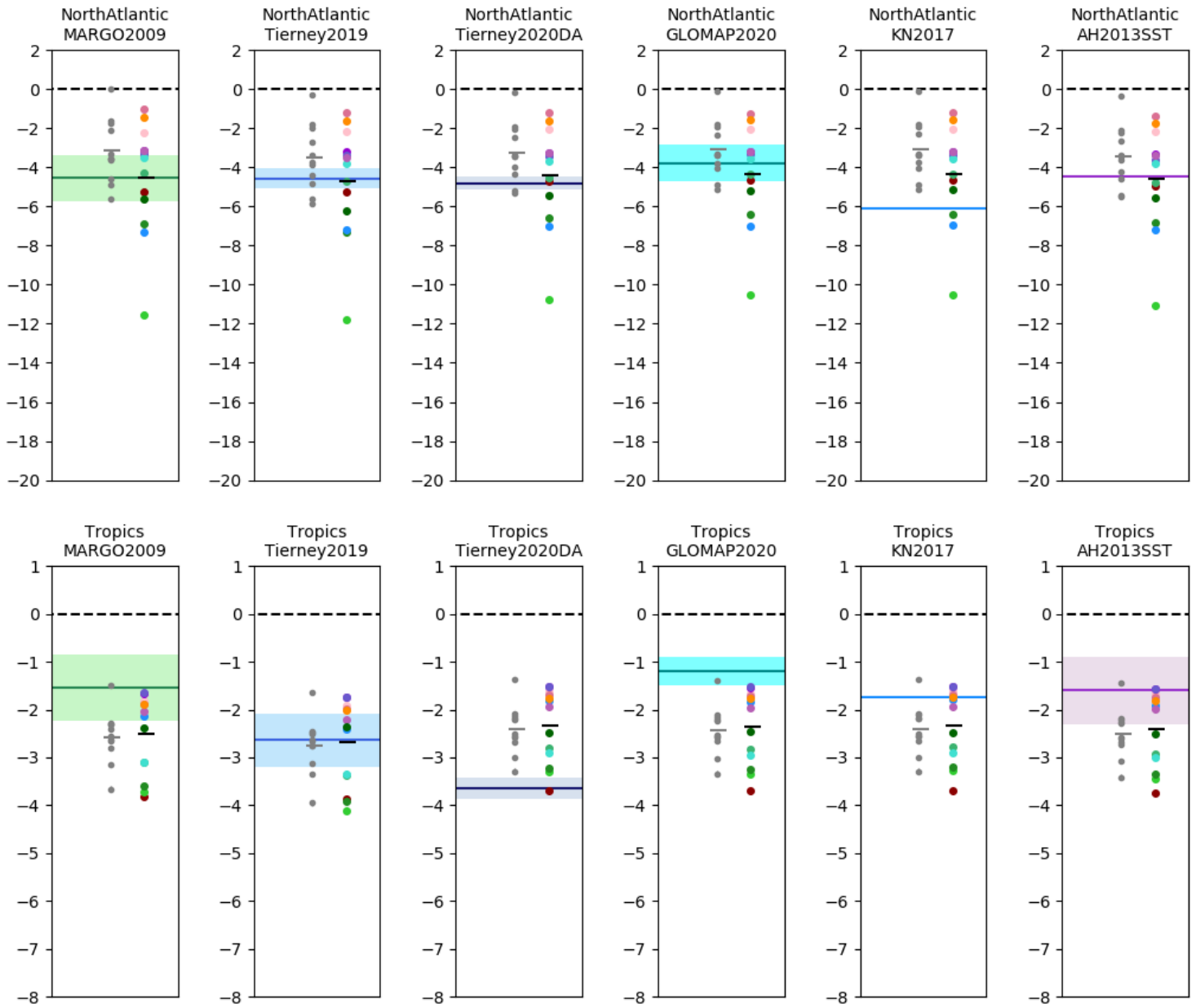

- PMIP3 mean

- PMIP4 mean

- MARGO 2009

Tierney et al., 2020

(w/o data assimilati

Tierney et al., 2020

(w/ data assimilatio

GLOMAP 2020,

* (Paul et al., 2020)

Kurahashi-Nakamur:

* et al., 2017

$\triangle$ Annan and Hargreav

+ PMIP3-CMIP5

AWI-ESM-1-1-LR

$\rightarrow$ AWI-ESM-2-1-LR

+ CESM1.2

- HadCM3-PMIP3

+ HadCM3-ICE6GC

+ HadCM3-GLAC1D

- ILOVECLIM-ICE-6G

+ iLOVECLIM-GLACID

+ INM-CM4-8

$\rightarrow$ IPSLCM5A2

$\rightarrow$ MIROC-ES2L

$\rightarrow$ MPI-ESM-1.2

+ UoT-CCSM4

\section{Supplementary Figure S3: model-data comparison over the North Atlantic and Tropical oceans.}


Table S1. Statistics on the LGM - PI mean annual surface air temperature anomalies, for the PMIP3 and PMIP4 ensembles. Units: ${ }^{\circ} \mathrm{C}$.

\begin{tabular}{|l|l|l|l|l|}
\hline PMIP phase & Region & Minimum & Average & Maximum \\
\hline PMIP3 & Globe & -5.72 & -4.71 & -2.70 \\
\hline PMIP4 & Globe & -7.17 & -4.77 & -3.26 \\
\hline PMIP3 & $\begin{array}{l}\text { Southern extratropics } \\
(\text { South of 30 } \mathrm{S})\end{array}$ & -5.79 & -3.82 & -2.42 \\
\hline PMIP4 & $\begin{array}{l}\text { Southern extratropics } \\
(\text { South of 30 } \mathrm{S})\end{array}$ & -8.15 & -4.77 & -1.27 \\
\hline PMIP3 & Tropics $\left(30^{\circ} \mathrm{S}-30^{\circ} \mathrm{N}\right)$ & -3.92 & -2.80 & -1.66 \\
\hline PMIP4 & Tropics $\left(30^{\circ} \mathrm{S}-30^{\circ} \mathrm{N}\right)$ & -4.0 & -2.68 & -1.77 \\
\hline PMIP3 & $\begin{array}{l}\text { Northern extratropics } \\
\left(\text { North of 30 } 30^{\circ} \mathrm{N}\right)\end{array}$ & -12.65 & -9.48 & -4.99 \\
\hline PMIP4 & $\begin{array}{l}\text { Northern extratropics } \\
\left(\text { North of 30 } 30^{\circ} \mathrm{N}\right)\end{array}$ & -12.58 & -8.83 & -5.18 \\
\hline
\end{tabular}


Table S2: Global and regional ( $30^{\circ}$ latitude bands) averages of the LGM - PI MAT anomalies over land and oceans, in ${ }^{\circ} \mathrm{C}$.

$\begin{array}{lcccccccc}\text { Model } & \text { PMIP phase } & \text { Globe } & 60-90^{\circ} \mathrm{N} & 30-60^{\circ} \mathrm{N} & 0-30^{\circ} \mathrm{N} & 30^{\circ} \mathrm{S}-0 & 30-60^{\circ} \mathrm{S} & 60-90 \mathrm{~S} \\ \text { CESM } & \text { PMIP3 } & -4.85 & -13.03 & -8.11 & -2.68 & -2.36 & -3.93 & -7.98 \\ \text { CNRM } & \text { PMIP3 } & -2.70 & -7.38 & -4.16 & -1.72 & -1.59 & -1.80 & -4.19 \\ \text { FGOALS-g2 } & \text { PMIP3 } & -4.63 & -14.78 & -8.59 & -3.32 & -2.72 & -1.89 & -4.21 \\ \text { GISSE2-p1 } & \text { PMIP3 } & -4.92 & -17.67 & -8.71 & -3.27 & -2.54 & -2.37 & -3.84 \\ \text { GISSE2-p2 } & \text { PMIP3 } & -5.19 & -18.87 & -9.13 & -3.32 & -2.68 & -2.61 & -4.22 \\ \text { IPSL } & \text { PMIP3 } & -4.64 & -11.37 & -7.96 & -3.62 & -2.98 & -2.35 & -5.55 \\ \text { MIROC } & \text { PMIP3 } & -5.40 & -17.11 & -11.17 & -2.74 & -2.16 & -2.74 & -9.04 \\ \text { MPI-p1 } & \text { PMIP3 } & -4.41 & -12.87 & -7.15 & -2.70 & -2.32 & -2.51 & -7.46 \\ \text { MPI-p2 } & \text { PMIP3 } & -4.67 & -13.94 & -7.79 & -2.88 & -2.42 & -2.51 & -7.57 \\ \text { MRI } & \text { PMIP3 } & -4.71 & -11.21 & -6.16 & -2.87 & -2.77 & -4.18 & -10.36 \\ \text { AWIESM1 } & \text { PMIP4 } & -3.75 & -10.20 & -6.02 & -1.79 & -1.74 & -2.75 & -8.27 \\ \text { AWIESM2 } & \text { PMIP4 } & -3.81 & -9.29 & -5.62 & -1.89 & -1.87 & -2.94 & -9.84 \\ \text { CESM1-2 } & \text { PMIP4 } & -6.80 & -16.98 & -9.09 & -3.96 & -4.05 & -6.51 & -12.82 \\ \text { HadCM3-PMIP3 } & \text { PMIP4 } & -7.16 & -18.69 & -11.96 & -4.32 & -3.76 & -5.16 & -11.06 \\ \text { HadCM3-ICE6GC } & \text { PMIP4 } & -5.92 & -16.97 & -9.32 & -3.60 & -3.20 & -4.08 & -9.27 \\ \text { HadCM3-GLAC1D } & \text { PMIP4 } & -6.46 & -16.52 & -9.32 & -3.91 & -3.77 & -5.47 & -10.82 \\ \text { iLOVECLIM-ICE-6G } & \text { PMIP4 } & -3.28 & -6.61 & -4.81 & -2.05 & -1.55 & -3.19 & -6.24 \\ \text { iLOVECLIM-GLAC1D } & \text { PMIP4 } & -3.26 & -6.70 & -4.71 & -2.03 & -1.54 & -3.16 & -6.29 \\ \text { INM-CM4-8 } & \text { PMIP4 } & -3.73 & -11.23 & -8.49 & -2.98 & -1.43 & -0.69 & -2.84 \\ \text { IPSLCM5A2 } & \text { PMIP4 } & -4.63 & -10.96 & -8.16 & -3.63 & -2.94 & -2.44 & -5.00 \\ \text { MIROC-ES2L } & \text { PMIP4 } & -4.02 & -12.44 & -7.78 & -2.44 & -1.87 & -2.19 & -5.35 \\ \text { MPI-PMIP4 } & \text { PMIP4 } & -3.90 & -10.33 & -6.30 & -2.22 & -1.89 & -2.73 & -7.54 \\ \text { UT-CCSM4 } & \text { PMIP4 } & -5.27 & -12.81 & -8.01 & -2.55 & -2.61 & -5.34 & -10.43\end{array}$


Table S3: Global and regional ( $30^{\circ}$ latitude bands) averages of the LGM - PI MAT anomalies over land, in ${ }^{\circ} \mathrm{C}$.

$\begin{array}{lcccccccc}\text { Model } & \text { PMIP phase } & \text { Globe } & 60-90^{\circ} \mathrm{N} & 30-60^{\circ} \mathrm{N} & 0-30^{\circ} \mathrm{N} & 30^{\circ} \mathrm{S}-0 & 30-60^{\circ} \mathrm{S} & 60-90 \mathrm{~S} \\ \text { CESM } & \text { PMIP3 } & -7.53 & -13.43 & -10.60 & -3.11 & -2.62 & -3.53 & -7.70 \\ \text { CNRM } & \text { PMIP3 } & -4.52 & -7.49 & -5.92 & -2.12 & -1.94 & -2.45 & -2.14 \\ \text { FGOALS-g2 } & \text { PMIP3 } & -7.27 & -14.39 & -10.21 & -4.01 & -3.28 & -3.21 & -4.61 \\ \text { GISSE2-p1 } & \text { PMIP3 } & -8.51 & -17.65 & -11.90 & -3.86 & -3.33 & -3.38 & -5.78 \\ \text { GISSE2-p2 } & \text { PMIP3 } & -8.67 & -18.86 & -11.67 & -3.86 & -3.46 & -3.57 & -6.10 \\ \text { IPSL } & \text { PMIP3 } & -7.55 & -12.26 & -10.72 & -3.95 & -3.30 & -3.59 & -6.42 \\ \text { MIROC } & \text { PMIP3 } & -9.74 & -16.23 & -14.02 & -3.20 & -2.73 & -2.98 & -5.16 \\ \text { MPI-p1 } & \text { PMIP3 } & -8.18 & -13.63 & -10.63 & -3.57 & -3.09 & -3.37 & -8.21 \\ \text { MPI-p2 } & \text { PMIP3 } & -8.71 & -15.01 & -11.41 & -3.82 & -3.20 & -3.22 & -8.34 \\ \text { MRI } & \text { PMIP3 } & -6.98 & -11.63 & -8.23 & -3.32 & -3.11 & -4.65 & -8.56 \\ \text { AWIESM1 } & \text { PMIP4 } & -6.62 & -11.85 & -8.76 & -1.83 & -1.60 & -2.33 & -7.63 \\ \text { AWIESM2 } & \text { PMIP4 } & -6.69 & -11.17 & -8.56 & -2.21 & -1.82 & -2.57 & -8.63 \\ \text { CESM1-2 } & \text { PMIP4 } & -9.49 & -16.61 & -12.19 & -4.75 & -4.31 & -6.38 & -10.22 \\ \text { HadCM3-PMIP3 } & \text { PMIP4 } & -11.84 & -18.65 & -14.66 & -5.83 & -4.95 & -5.70 & -7.44 \\ \text { HadCM3-ICE6GC } & \text { PMIP4 } & -10.10 & -17.60 & -12.62 & -4.68 & -4.43 & -4.66 & -10.60 \\ \text { HadCM3-GLAC1D } & \text { PMIP4 } & -10.40 & -17.01 & -12.12 & -5.16 & -4.82 & -5.49 & -6.13 \\ \text { iLOVECLIM-ICE-6G } & \text { PMIP4 } & -4.67 & -7.83 & -5.67 & -2.53 & -1.71 & -3.60 & -5.60 \\ \text { iLOVECLIM-GLAC1D } & \text { PMIP4 } & -4.80 & -7.70 & -5.51 & -2.51 & -1.71 & -3.72 & -5.16 \\ \text { INM-CM4-8 } & \text { PMIP4 } & -7.33 & -12.95 & -11.42 & -3.58 & -1.83 & -2.45 & -3.98 \\ \text { IPSLCM5A2 } & \text { PMIP4 } & -7.60 & -11.71 & -10.96 & -4.07 & -3.55 & -3.77 & -6.01 \\ \text { MIROC-ES2L } & \text { PMIP4 } & -6.88 & -12.69 & -10.44 & -2.94 & -1.89 & -2.85 & -5.50 \\ \text { MPI-PMIP4 } & \text { PMIP4 } & -7.15 & -11.72 & -9.37 & -2.87 & -2.28 & -3.34 & -5.63 \\ \text { UT-CCSM4 } & \text { PMIP4 } & -7.10 & -13.45 & -9.59 & -2.58 & -2.47 & -4.30 & -9.09\end{array}$


Table S4: Global and regional ( $30^{\circ}$ latitude bands) averages of the LGM - PI MAT anomalies over oceans, in ${ }^{\circ} \mathrm{C}$.

$\begin{array}{lcccccccc}\text { Model } & \text { PMIP phase } & \text { Globe } & 60-90^{\circ} \mathrm{N} & 30-60^{\circ} \mathrm{N} & 0-30^{\circ} \mathrm{N} & 30^{\circ} \mathrm{S}-0 & 30-60^{\circ} \mathrm{S} & 60-90 \mathrm{~S} \\ \text { CESM } & \text { PMIP3 } & -2.79 & -12.14 & -3.98 & -2.30 & -2.17 & -4.04 & -6.71 \\ \text { CNRM } & \text { PMIP3 } & -1.25 & -6.77 & -1.19 & -1.44 & -1.39 & -1.79 & -2.64 \\ \text { FGOALS-g2 } & \text { PMIP3 } & -2.42 & -15.71 & -4.98 & -2.82 & -2.39 & -1.88 & -1.35 \\ \text { GISSE2-p1 } & \text { PMIP3 } & -2.62 & -17.59 & -4.15 & -2.97 & -2.26 & -2.36 & -2.08 \\ \text { GISSE2-p2 } & \text { PMIP3 } & -2.95 & -18.78 & -5.36 & -3.06 & -2.40 & -2.61 & -2.50 \\ \text { IPSL } & \text { PMIP3 } & -2.55 & -9.59 & -3.56 & -3.33 & -2.83 & -2.33 & -2.74 \\ \text { MIROC } & \text { PMIP3 } & -2.51 & -19.09 & -6.15 & -2.47 & -1.96 & -2.80 & -4.88 \\ \text { MPI-p1 } & \text { PMIP3 } & -2.14 & -10.01 & -2.49 & -2.29 & -2.03 & -2.50 & -5.29 \\ \text { MPI-p2 } & \text { PMIP3 } & -2.27 & -10.24 & -2.96 & -2.43 & -2.12 & -2.51 & -5.37 \\ \text { MRI } & \text { PMIP3 } & -2.83 & -10.41 & -2.49 & -2.54 & -2.57 & -4.25 & -8.77 \\ \text { AWIESM1 } & \text { PMIP4 } & -1.95 & -4.59 & -2.39 & -1.74 & -1.71 & -2.84 & -7.02 \\ \text { AWIESM2 } & \text { PMIP4 } & -2.01 & -3.32 & -1.80 & -1.74 & -1.81 & -3.02 & -8.88 \\ \text { CESM1-2 } & \text { PMIP4 } & -4.96 & -17.42 & -4.60 & -3.55 & -3.92 & -6.60 & -14.02 \\ \text { HadCM3-PMIP3 } & \text { PMIP4 } & -4.08 & -17.68 & -7.78 & -3.56 & -3.31 & -5.18 & -8.40 \\ \text { HadCM3-ICE6GC } & \text { PMIP4 } & -3.46 & -14.73 & -4.81 & -3.06 & -2.77 & -4.08 & -7.05 \\ \text { HadCM3-GLAC1D } & \text { PMIP4 } & -3.91 & -14.29 & -5.40 & -3.28 & -3.38 & -5.53 & -9.04 \\ \text { iLOVECLIM-ICE-6G } & \text { PMIP4 } & -2.05 & -7.50 & -2.86 & -1.76 & -1.44 & -3.23 & -5.74 \\ \text { iLOVECLIM-GLAC1D } & \text { PMIP4 } & -1.91 & -8.12 & -2.80 & -1.73 & -1.43 & -3.18 & -5.08 \\ \text { INM-CM4-8 } & \text { PMIP4 } & -1.34 & -7.68 & -4.32 & -2.61 & -1.22 & -0.60 & 0.22 \\ \text { IPSLCM5A2 } & \text { PMIP4 } & -2.53 & -9.40 & -3.73 & -3.29 & -2.71 & -2.42 & -2.06 \\ \text { MIROC-ES2L } & \text { PMIP4 } & -2.20 & -12.46 & -3.88 & -2.19 & -1.84 & -2.20 & -3.71 \\ \text { MPI-PMIP4 } & \text { PMIP4 } & -1.88 & -5.77 & -2.22 & -1.90 & -1.71 & -2.74 & -6.13 \\ \text { UT-CCSM4 } & \text { PMIP4 } & -3.74 & -11.94 & -5.11 & -2.42 & -2.60 & -5.50 & -9.97\end{array}$

